\title{
Topological phases in the Neuberger-Dirac operator
}

\author{
Ting-Wai Chiu* \\ Department of Physics, National Taiwan University, Taipei, Taiwan 106, Republic of China
}

(Received 11 November 1998; published 11 November 1999)

\begin{abstract}
The response of the Neuberger-Dirac fermion operator $D=1+V$ in the topologically nontrivial background gauge field depends on the negative mass parameter $m_{0}$ in the Wilson-Dirac fermion operator $D_{w}$, which enters $D$ through the unitary operator $V=D_{w}\left(D_{w}^{\dagger} D_{w}\right)^{-1 / 2}$. We classify the topological phases of $D$ by comparing its index to the topological charge of the smooth background gauge field. An exact discrete symmetry in the topological phase diagram is proved for any gauge configurations. A formula for the index of $D$ in each topological phase is derived by obtaining the total chiral charge of the zero modes in the exact solution of the free fermion propagator. [S0556-2821(99)06521-2]

PACS number(s): 11.15.Ha, 11.30.Fs, 11.30.Rd
\end{abstract}

\section{INTRODUCTION}

It is well known that the Neuberger-Dirac operator [1,2]

$$
D=1+V, \quad V=D_{w}\left(D_{w}^{\dagger} D_{w}\right)^{-1 / 2}
$$

reproduces the exact zero modes with definite chirality and realizes the Atiyah-Singer index theorem on a finite lattice for smooth background gauge fields with a nonzero topological charge [3]. However, this is only true for the negative mass parameter $m_{0}$ approximately in the range $\left(0,2 r_{w}\right)$ for the Wilson-Dirac fermion operator $D_{w}$ with Wilson parameter $r_{w}>0$,

$$
D_{w}=-m_{0}+\frac{1}{2}\left[\gamma_{\mu}\left(\nabla_{\mu}^{*}+\nabla_{\mu}\right)-r_{w} \nabla_{\mu}^{*} \nabla_{\mu}\right] \text {, }
$$

where $\nabla_{\mu}$ and $\nabla_{\mu}^{*}$ are the forward and backward difference operators defined in the following:

$$
\begin{aligned}
& \nabla_{\mu} \psi(x)=U_{\mu}(x) \psi(x+\hat{\mu})-\psi(x), \\
& \nabla_{\mu}^{*} \psi(x)=\psi(x)-U_{\mu}^{\dagger}(x-\hat{\mu}) \psi(x-\hat{\mu}) .
\end{aligned}
$$

Except for the region $m_{0}<0$, which corresponds to the positive mass in $D_{w}$ that gives the zero index of $D$ for any background gauge field, as been noted by Neuberger in Ref. [2], there is literally no discussion in the literature about what would happen to $D$ for other values of $m_{0}$, namely, $m_{0}$ $\geqslant 2 r_{w}$. In Ref. [4], it is remarked that $D_{w}$ must have an appropriately tuned negative mass term such that $D$ is in the right phase to reproduce QCD. However, there is no further discourse on how to identify the phase such that $D$ can possess the proper topological characteristics and reproduce the correct continuum physics.

In this paper we investigate the topological phases of $D$ with respect to the negative mass parameter $m_{0}$ and the background gauge field, respectively, by comparing its index $(D)$ to the topological charge $Q$ of the background gauge field. If index $(D)=0$ for any smooth gauge configurations, then $D$ is said to be in the topologically trivial phase. If index $(D)$ $=Q$, then $D$ is said to be in the topologically proper phase. If $D$ is not topologically trivial, but $\operatorname{index}(D) \neq Q$, then $D$ is said to be in the topologically improper phase.

First, we sketch schematically the topological phases of $D$ in Fig. 1. On a $d$-dimensional lattice, ${ }^{1}$ the topological phases of $D$ in the free fermion limit divide $m_{0}$ values into $d+2$ intervals according to the real eigenmodes of $D$. As we turn on the background gauge field, the locations of the phase boundaries also evolve accordingly. The first region cuts the interval $m_{0}<0$ in the free fermion limit. In this phase, the spectrum of $D$ does not possess any zero modes and thus cannot describe massless particles, and the index is zero for any background gauge field. Therefore $D$ is called topologically trivial in this phase. The second region cuts the interval $0<m_{0}<2 r_{w}$ in the free fermion limit. In this phase, $D$ has exact zero modes with definite chirality, and the index of $D$ is equal to the topological charge $Q_{\text {top }}$ of the background gauge field. Therefore $D$ is called topologically proper in this phase. The third region cuts the interval $2 r_{w}<m_{0}$ $<4 r_{w}$ in the free field limit. In this phase, $D$ also has exact zero modes with definite chirality, but the index of $D$ is (1 $-d) Q_{\text {top }}$, not equal to the topological charge of the background gauge field. Thus $D$ is called topologically improper in this phase. The next phase again has width $2 r_{w}$ in the free fermion limit. The process of dividing $m_{0}$ into different topological phases continues until it reaches $m_{0}=2 d r_{w}$ in the free fermion limit, then the last phase is the region with the edge, $2 d r_{w}<m_{0}<\infty$. In any dimensions, only the region cutting the interval $0<m_{0}<2 r_{w}$ in the free fermion limit is the topologically proper phase for $D$ : other phases are either topologically trivial or improper. The index along each one of the phase boundaries starting at $\left(m_{0}=2 k r_{w}, k=0, \ldots, d\right)$ is zero identically for any background gauge field.

In the free fermion limit, we can distinguish different phases of $D$ according to its real eigenmodes. The phase transition is signaled by the change of the numbers of real eigenmodes. We note that in the free fermion limit the real eigenmodes of $D$ must be either at the origin or the corners

\footnotetext{
${ }^{1}$ In this paper we always assume that $d$ is a positive even integer.
}

*Email address: twchiu@phys.ntu.edu.tw 


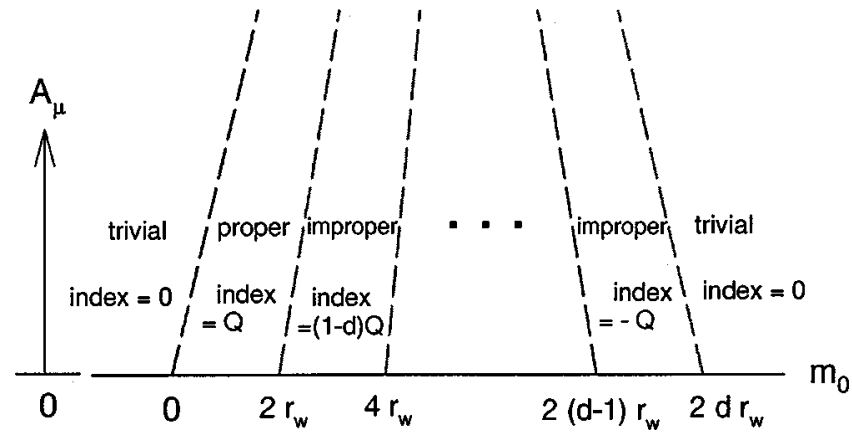

FIG. 1. The topological phase diagram of $D$ on a $d$-dimensional lattice for a smooth background gauge field. The phase boundaries denoted by dashed lines are sketched to indicate their evolutions with respect to the background gauge field. The critical points in the free fermion limit (at the horizontal line) are determined exactly. The reflection symmetry with respect to the symmetry axis $m_{0}$ $=d r_{w}$ is also shown explicitly.

of the Brillouin zone. However, the converse of this statement is not always true, since at some values of $m_{0}$, some of the eigenmodes at the corners of the Brillouin zone become complex. Then a formula for the index of $D$ in each phase can be derived (in Sec. III) by obtaining the total chiral charge of the zero modes in the exact solution of the free fermion propagator. For smooth background gauge fields, this formula (34) gives the correct index in each phase except at the phase boundaries $\left(m_{0}=2 k r_{w}, k=1, \ldots, d\right)$.

For any arbitrary gauge configuration, we prove that there exists an exact reflection symmetry in the topological phase diagram with respect to the symmetry axis $m_{0}=d r_{w}$, i.e., index $\left[D\left(m_{0}\right)\right]=-\operatorname{index}\left[D\left(2 d r_{w}-m_{0}\right)\right]$, for a finite lattice of even number of sites in each dimension. It follows that if there is a phase boundary located at $m_{0}=x<d r_{w}$ on the left-hand side (LHS), then there is another phase boundary located at $m_{0}=2 d r_{w}-x$ on the RHS, and these two phase boundaries appear as images to each other with respect to the mirror at $m_{0}=d r_{w}$. Since $d$ is an even integer, the exact reflection symmetry implies that the symmetry axis $m_{0}$ $=d r_{w}$ is a phase boundary with zero index and its location is invariant for any background gauge configurations (for example, see Figs. 2 and 3).

As we turn on the background gauge field smoothly from zero to some finite values, the locations of the phase boundaries also evolve accordingly. When the gauge fields reach a certain level of magnitude and roughness, the phase boundaries bifurcate and the phase diagram becomes very complicated. We expect that for gauge configurations in dynamical simulations the phase boundaries may change significantly from those of smooth background gauge fields. However, the exact reflection symmetry must hold in general and provides a useful scheme to identify the topological phases especially for rough gauge configurations. We note that at one of the topological phase boundaries of $D$, the theory is free of species doublings in the free fermion limit, but turns out to be topologically trivial in any background gauge fields. This provides an explicit example that for $D$ free of species doubling in the free fermion limit is not sufficient to guarantee the realization of the Atiyah-Singer index theorem on the lattice, in contrast to the condition of reproducing correct axial anomaly in perturbation calculations [5]. This may indicate that the topological characteristics of the fermion operator $D$ are of nonperturbative origin and thus could not be revealed by any perturbation calculations. We note in passing that the locality of the Ginsparg-Wilson fermion operator $D$ is not relevant to its zero modes and index, as discussed and demonstrated in Refs. [6,7].

The outline of this paper is as follows. In Sec. II, we prove the exact reflection symmetry in the topological phase diagram of $D$. In Sec. III, we obtain exact solutions of the eigenvalues and fermion propagator of $D$ in the free fermion limit, then use them to analyze the topological phases of $D$. A formula for the index of $D$ in each phase is derived. In Sec. IV, we perform numerical experiments to investiagate the topological phase diagrams of $D$ in two- and fourdimensional lattices, respectively. In Sec. V, we summarize and also demonstrate the bifurcation of topological phases by increasing the amplitudes of the local sinusoidal fluctuations of the background gauge field on a two-dimensional lattice. In the Appendix, we discuss the spectral flow of the Neuberger operator as a function of the negative mass parameter $m_{0}$, in particular its behaviors at the phase boundaries, in order to gain a perspective on the underlying mechanism which gives a zero index at the phase boundaries.

\section{DISCRETE SYMMETRY OF THE INDEX}

In this section, we prove that for a finite lattice with an even number of sites in each dimension $\left(L_{\mu}=N_{\mu} a, N_{\mu}\right.$ $=$ even integer, $\mu=1, \ldots, d)$, the topological phase diagram of the Neuberger-Dirac operator $D\left(m_{0}\right)$ has an exact reflection symmetry with respect to the axis $m_{0}=d r_{w}$, and the index of $D$ is odd under the reflection, i.e., index $\left[D\left(m_{0}\right)\right]=$ - index $\left[D\left(2 d r_{w}-m_{0}\right)\right]$.

Proof. For a given gauge configuration, the index of $D\left(m_{0}\right)$ is defined to be $n_{-}\left(m_{0}\right)-n_{+}\left(m_{0}\right)$, where $n_{-}\left(n_{+}\right)$ denotes the number of zero modes of negative (positive) chirality. Let $\phi_{+}^{s}, s=1, \ldots, n_{+}$, be the normalized eigenfunctions of the zero modes with positive chirality:

$$
D\left(m_{0}\right) \phi_{+}^{s}=0, \quad \gamma_{5} \phi_{+}^{s}=\phi_{+}^{s} .
$$

Then $\phi_{+}^{s}$ is a real eigenmode of $D_{w}$ with negative real eigenvalue $-\mu_{s}$ such that

$$
\begin{gathered}
V\left(m_{0}\right) \phi_{+}^{s}=-\phi_{+}^{s}, \\
D_{w}\left(m_{0}\right) \phi_{+}^{s}=-\mu_{s} \phi_{+}^{s} .
\end{gathered}
$$

Similar equations for zero modes with negative chirality are understood. The standard Wilson-Dirac lattice fermion operator $D_{w}$, Eq. (2), can be rewritten as

$$
\left[D_{w}\left(m_{0}\right)\right]_{x, y}=\left(d r_{w}-m_{0}\right) \delta_{x, y}-\left[T\left(r_{w}\right)\right]_{x, y},
$$

where 


$$
\begin{aligned}
{\left[T\left(r_{w}\right)\right]_{x, y}=} & \frac{1}{2} \sum_{\mu}\left[\left(r_{w}-\gamma_{\mu}\right) U_{\mu}(x) \delta_{x+\hat{\mu}, y}\right. \\
& \left.+\left(r_{w}+\gamma_{\mu}\right) U_{\mu}^{\dagger}(x-\hat{\mu}) \delta_{x-\hat{\mu}, y}\right] .
\end{aligned}
$$

The Dirac and color indices are suppressed. The fundamental properties of $T$ are [8]

$$
\begin{aligned}
\gamma_{5} T \gamma_{5} & =T^{\dagger}, \\
S T S & =-T,
\end{aligned}
$$

where $S_{x, y}=\Pi_{\mu}(-1)^{x_{\mu} / a} \delta_{x, y}$ which satisfies $S^{2}=1$ and $S^{\dagger}$ $=S$. The first property is the usual Hermiticity condition. The second property is also obvious except at the boundaries of the lattice (with periodic boundary conditions), where the forward and backward difference operators in $T$ would produce a factor $+1(-1)$ for an odd (even) number of sites in this direction. Therefore Eq. (9) is satisfied exactly on a finite lattice having an even number of sites in each dimension, but only satisfied approximately on a finite lattice having an odd number of sites in some dimensions. Of course, it is satisfied exactly on the infinite lattice. It is evident that an eigenfunction of $D_{w}$ must be an eigenfunction of $T$, and vice versa. Then, from Eqs. (5) and (6), we have

$$
T \phi_{+}^{s}=t_{s} \phi_{+}^{s},
$$

where $t_{s}=\mu_{s}+\left(d r_{w}-m_{0}\right)$. Using Eq. (9) and $S^{2}=1$, we obtain

$$
\begin{gathered}
\operatorname{det}(T-t \rrbracket)=\operatorname{det}(S) \operatorname{det}(T-t \Perp) \operatorname{det}(S) \\
\quad=\operatorname{det}(S T S-t \Perp)=\operatorname{det}(-T-t \Perp) .
\end{gathered}
$$

Thus the eigenvalues of $T$ must come in $\pm t_{s}$ pairs. It follows that the eigenvalues of $D_{w}\left(m_{0}\right)$ must come in $d r_{w}-m_{0} \pm t_{s}$ pairs. Now, if we substitute $m_{0}$ by $2 d r_{w}-m_{0}$, then the eigenvalues of $D_{w}\left(2 d r_{w}-m_{0}\right)$ come in $-\left(d r_{w}-m_{0} \mp t_{s}\right)$ pairs. Therefore, from Eq. (5), if $D_{w}\left(m_{0}\right)$ has a negative real eigenvalue $-\mu_{s}$ for the eigenfunction $\phi_{+}^{s}$, then $D_{w}\left(2 d r_{w}\right.$ $-m_{0}$ ) has a corresponding positive real eigenvalue $+\mu_{s}$ with the same eigenfunction $\phi_{+}^{s}$, and this implies that $D\left(2 d r_{w}-m_{0}\right)$ has a +2 real eigenmode while $D\left(m_{0}\right)$ has a zero mode, and vice versa, i.e.,

$$
\begin{aligned}
& n_{2}^{+}\left(m_{0}\right)=n_{+}\left(2 d r_{w}-m_{0}\right), \\
& n_{2}^{-}\left(m_{0}\right)=n_{-}\left(2 d r_{w}-m_{0}\right), \\
& n_{+}\left(m_{0}\right)=n_{2}^{+}\left(2 d r_{w}-m_{0}\right), \\
& n_{-}\left(m_{0}\right)=n_{2}^{-}\left(2 d r_{w}-m_{0}\right) .
\end{aligned}
$$

As it was first proved in Ref. [3] that the number of zero modes and the number of +2 modes for $D\left(m_{0}\right)$ must obey the relation

$$
n_{2}^{+}\left(m_{0}\right)-n_{2}^{-}\left(m_{0}\right)=n_{-}\left(m_{0}\right)-n_{+}\left(m_{0}\right),
$$

we immediately obtain

$$
n_{+}\left(2 d r_{w}-m_{0}\right)-n_{-}\left(2 d r_{w}-m_{0}\right)=n_{-}\left(m_{0}\right)-n_{+}\left(m_{0}\right) \text {. }
$$

That is,

$$
\operatorname{index}\left[D\left(2 d r_{w}-m_{0}\right)\right]=-\operatorname{index}\left[D\left(m_{0}\right)\right] \text {. }
$$

This is the discrete symmetry of index(D) which holds for any gauge configuration. It transcribes to the reflection symmetry in the topological phase diagram with the symmetry axis at $m_{0}=d r_{w}$. When the gauge fields are very rough, the topological phase diagram becomes very complicated; the only thing that surely survives in such chaotic environments is the exact discrete symmetry, Eq. (17). For a finite lattice having an odd number of sites in some directions, the reflection symmetry Eq. (17), is only an approximate discrete symmetry which tends to the exact reflection symmetry in the infinite lattice limit. One immediate consequence of the exact discrete symmetry (17) is that at the symmetry axis $m_{0}$ $=d r_{w}$, the index is zero for any background gauge field:

$$
\text { index }\left[D\left(d r_{w}\right)\right]=0 \text {. }
$$

Since $d$ is an even integer, the symmetry axis $m_{0}=d r_{w}$ is a phase boundary with zero index and its location is invariant for any background gauge configuration (for example, see Figs. 2 and 3).

We also note that $T\left(r_{w}\right)$ has another discrete symmetry,

$$
\left[T\left(r_{w}\right)\right]^{\dagger}=-T\left(-r_{w}\right)
$$

which follows directly from Eq. (7). Then we have the following discrete symmetry for $D_{w}$ :

$$
\left[D_{w}\left(m_{0}, r_{w}\right)\right]^{\dagger}=-D_{w}\left(-m_{0},-r_{w}\right) .
$$

From this relation, we immediately know that the zero modes of $D\left(m_{0}, r_{w}\right)$ are the +2 eigenmodes of $D\left(-m_{0}\right.$, $-r_{w}$ ), and vice versa. Using Eq. (15), we obtain the following discrete symmetry for the index of $D$ :

$$
\text { index }\left[D\left(m_{0}, r_{w}\right)\right]=-\operatorname{index}\left[D\left(-m_{0},-r_{w}\right)\right] \text {. }
$$

This discrete symmetry implies that if we change the sign of the Wilson parameter $r_{w}$, then the new topological phase diagram is the negative image of the old topological phase diagram with respect to the mirror at $m_{0}=0$. This reflection symmetry will not be exploited in this paper.

We remark that the discrete symmetries, Eqs. (17) and (21), discussed above also hold for the generalized Neuberger-Dirac operator [6]

$$
D=2 M(1+V)[(1-V)+2 M R(1+V)]^{-1},
$$

where $R$ is a nonsingular Hermitian local operator which is trivial in the Dirac space, $M$ is an arbitrary mass scale, and $V\left(m_{0}, r_{w}\right)$ is the unitary operator defined in Eq. (1). The reason for these discrete symmetries holding for the Ginsparg-Wilson generalized $D$ in Eq. (22) is that the zero modes of $D$ are invariant for any $R[6,7]$, and hence any 
relationships involving only the indices must be $R$ invariant. The proof for the general case is obvious from the above proof.

\section{EXACT SOLUTIONS IN THE FREE FERMION LIMIT}

In the free fermion limit, the index of $D$ must be zero, but we can use the number of zero modes of $D$ to distinguish different topological phases of $D$. Since there is a symmetry between the number of zero modes and the number of +2 modes as first shown in Ref. [3], Eqs. (11)-(14), then it suffices to use both the number of zero modes and the number of +2 eigenmodes as the order parameter for the topological phases of $D$ in the free fermion limit. Then a phase transition is signaled by any changes of these two numbers. Alternatively, we can examine the fermion propagator and use the number of massless (primary and doubled) fermion modes it contains to distinguish different topological phases of $D$. From the total chiral charge $Q_{5}$ of the massless fermion modes in the fermion propagator, we can determine the integer multiplier of the $F \widetilde{F}$ term in the axial anomaly $q(x ; A, D)=\operatorname{tr}\left[\gamma_{5}(R D)(x, x)\right]$ for $D$ in a smooth background gauge field of topological charge $Q_{\text {top }}$, which also can be determined by explicit perturbation calculations. Furthermore, we can also predict that the index of $D$ is equal to the total chiral charge times the topological charge of the background gauge field, i.e.,

$$
\operatorname{index}(D)=Q_{5} Q_{\text {top }} .
$$

This expression is deduced straightforwardly from weak coupling perturbation theory, which in fact correctly predicts the index of $D$ in smooth background gauge fields for all values of $m_{0}$ except at the phase boundaries starting at $m_{0}$ $=2 r_{w}, 4 r_{w}, \ldots, 2 d r_{w}$, where the index is always zero regardless of the total chiral charge of the zero modes. The disagreement between the prediction (23) and the zero index at the phase boundaries indicates that there may be some nonperturbative or topological effects which cannot be taken into account in the perturbation theory. We will show that Eq. (23) violates the exact reflection symmetry at the phase boundaries.

First, we obtain exact solutions of the eigenvalues and fermion propagator of $D$. In the free fermion limit, the eigenvalues of the Neuberger-Dirac operator $D[\mathrm{Eq}$. (1)] on a $d$-dimensional lattice $\left[L_{\mu}=N_{\mu} a, \mu=1, \ldots, d\right.$ (even integer) with periodic boundary conditions are

$$
\lambda_{s}=1+\frac{u(p)}{N(p)} \pm i \frac{\sqrt{\sum_{\mu=1}^{d} \sin ^{2}\left(p_{\mu} a\right)}}{N(p)},
$$

where

$$
\begin{gathered}
u(p)=-m_{0}+r_{w} \sum_{\mu=1}^{d}\left[1-\cos \left(p_{\mu} a\right)\right] \\
N(p)=\sqrt{u^{2}(p)+\sum_{\mu=1}^{d} \sin ^{2}\left(p_{\mu} a\right)},
\end{gathered}
$$

and each real $\lambda_{s}$ has degeneracy $2^{[d / 2]}$ consisting of chirality \pm 1 pairs in the Dirac space, while each complex $\lambda_{s}$ has degeneracy $2^{[(d-2) / 2]}$.

The free fermion propagator in momentum space is [3]

$$
S_{F}^{(0)}(p)=\frac{a}{2} 1+\frac{a}{i \gamma_{\mu} \sin \left(p_{\mu} a\right)} T(p),
$$

where

$$
T(p)=\frac{1}{2}[N(p)-u(p)] .
$$

The constant term $a / 2$, which vanishes in the continuum limit, can be ignored since its role is the same as the chirality breaking operator $R$ in the Ginsparg-Wilson fermion propagator.

The second term is equal to the naive lattice fermion propagator times the factor $T(p)$. The suppression of the doubled modes is due to the last factor $T(p)$, which becomes zero for some or all of the doubled modes depending on the value of $m_{0}$. We have deliberately written the fermion propagator in the form of Eq. (28) such that the decoupling mechanism for the doubled modes is the most appealing to us. It is interesting to note that the way doubled modes are decoupled in Eq. (28) is very different from that of the Wilson fermion propagator. The former is a complete suppression even at finite lattice spacing, e.g., $T(p)=0$ for all doubled modes for $0<m_{0} \leqslant 2 r_{w}$, while the latter is of order $a$, which is completely decoupled only in the continuum limit $(a \rightarrow 0)$. This implies that the Neuberger-Dirac fermion can reproduce the correct continuum axial anomaly and the exact Atiyah-Singer index theorem at finite lattice spacing, while the Wilson fermion can only recover them in the continuum limit. In other words, any (primary or doubled) massless fermion mode in the Neuberger-Dirac fermion propagator emerges in a very clean-cut way, i.e., either existing or vanishing, for any finite lattice spacing. 
In order to discuss the chiral charge of the doubled modes, we consider the free fermion propagator in the continuum limit $(a \rightarrow 0)$ on the infinite lattice with the momentum space, the Brillouin zone (BZ) which is specified by $p_{\mu} \in(-\pi / a, \pi / a]$ for $\mu=1, \ldots, d$. In the vicinity of the origin $p=0$, we can expand $\sin \left(p_{\mu} a\right)=p_{\mu} a+O\left(a^{2} p_{\mu}^{2}\right)$ and $T(p)$ $=m_{0}+O\left(a p^{2}\right)$ in Eq. (28) to obtain

$$
S_{F}^{(0)}(p) \stackrel{a, p \rightarrow 0}{\longrightarrow}-\frac{i \gamma_{\mu} p_{\mu}}{p^{2}} m_{0}+O\left(a^{2} p\right)
$$

where the constant term $a / 2$ has been subtracted and the factor $m_{0}$ can be absorbed by using the generalized Dirac operator (22) with $M=m_{0}$ and $R=1 / 2 m_{0}$ : thus, $D=m_{0}(1$ $+V)$. Then $S_{F}^{(0)}(p)$ in Eq. (30) agrees with the free fermion propagator in continuum. This is the primary mode of the lattice fermion. In addition to the primary mode, it is also possible for $S_{F}^{(0)}(p)$ to develop massless doubled modes at other regions of the $\mathrm{BZ}$ due to the presence of $\sin \left(p_{\mu} a\right)$ rather than $p_{\mu} a$ in Eq. (28). It is evident that the massless doubled modes can only occur around the $2^{d}-1$ corners of the BZ, i.e., $\otimes_{\mu=1}^{d}\{0, \pi / a\}-\{p=0\}$. If the factor $T(p)$ vanishes smoothly at any one of these corners, then the doubled mode at this corner is decoupled from the theory: otherwise, this doubled mode will contribute to the propagator. Furthermore, a doubled mode may possess an opposite chiral charge of the primary mode due to the property of $\sin \left(p_{\mu} a\right)$. When $p_{\mu}$ is near $\pi / a$, we must shift the momentum $p_{\mu}$ to $p_{\mu}^{\prime}$ $=p_{\mu}-\pi / a$ such that the higher order terms $O\left(p^{2} a^{2}\right)$ in $\sin \left(p_{\mu}^{\prime} a\right)$ can be neglected in the continuum limit $a \rightarrow 0$. Then the relationship $\sin \left(p_{\mu} a\right)=-\sin \left(p_{\mu}^{\prime} a\right)$ produces an extra minus sign which can be absorbed by redefining $\gamma_{\mu}^{\prime}=-\gamma_{\mu}$. Since $\gamma_{5}=\prod_{\mu=1}^{d} \gamma_{\mu}$, the effective $\gamma_{5}^{\prime}$ for a doubled mode is equal to $+\gamma_{5}$ or $-\gamma_{5}$ depending on whether the number of $\pi / a$ momentum components is even or odd. Since the sign of $\gamma_{5}$ is usually taken to be the sign of the chiral charge, then we have $2^{d-1}-1$ doubled modes of positive chiral charge, but $2^{d-1}$ of negative chiral charge. After taking into account the primary mode, the number of fermion modes of positive chiral charge is equal to the number of negative ones. If all these modes contribute to the propagator, then the total chiral charge vanishes, the axial anomaly cancels, and the index is zero in any background gauge field. That is the case for the naive lattice fermion.

For the Neuberger operator, the suppression of doubled modes depends on the factor $T(p)$, which is a function of $m_{0}$. At the origin and the corners of the BZ, $\sin \left(p_{\mu} a\right)=0$; then, $T(p)$ can be simplified to

$$
T(p)=\frac{1}{2}[|u(p)|-u(p)],
$$

where the possible values of $u(p)$ are the following:

$$
u(p)=-m_{0},-m_{0}+2 r_{w},-m_{0}+4 r_{w}, \ldots,-m_{0}+2 d r_{w}
$$

for these $2^{d}$ momenta. From Eq. (31), it is evident that $T(p)=0$ for $u(p) \geqslant 0$.

For $m_{0} \leqslant 0, T(p)=0$ for all $2^{d}$ massless fermion modes: hence, they are all suppressed and decoupled from the theory. Since the theory does not have any massless fermion modes, the index must be zero after the background gauge field is turned on. This is a topologically trivial phase of $D$. Note that for $m_{0} \leqslant 0, D$ can be shown to be topologically trivial for any background gauge field since $D_{w}$ does not possess any negative eigenvalue for a positive mass parameter.

For all values of $m_{0}>0$, the primary mode at $p=0$ is recovered to the theory since $u(0)<0$ and $T(0) \neq 0$. For 0 $<m_{0}<2 r_{w}, u(p)>0$ and $T(p)=0$ for all doubled modes, so all doubled modes are still decoupled from the theory. When we turn on the background gauge field with topological charge $Q_{\text {top }}$, the primary mode then gives an index equal to $+Q_{\text {top }}$, the same as the continuum theory. This is the topologically proper phase of $D$.

At the phase boundary $m_{0}=2 r_{w}$, all doubled modes have $T(p)=0$; hence, they are decoupled from the theory. However, when we turn on the background gauge field with topological charge $Q_{\text {top }}$, the index is always zero. From the viewpoint of perturbation theory, it is not clear what has happened. If we examine the doubled modes more closely, we find that there are $d$ doubled modes which are just "critically" decoupled, i.e., each having only one nonzero $(\pi / a)$ momentum component which gives $u(p)=0$ and $T(p)=0$; hence, they are decoupled from the theory. On the other hand, if we assume that these $d$ doubled modes still remain in the theory, then the index should be $(1-d) Q_{\text {top }}$ rather than zero, since they carry negative chiral charge. So this assumption cannot be true. Moreover, $T(p)$ goes to zero smoothly at these $d$ double modes. Hence the theory is well behaved and free of massless doublers at the phase boundary $m_{0}=2 r_{w}$ in the free fermion limit, but becomes topologically trivial in any background gauge field. This "anomalous" phenomenon seems to be of nonperturbative or topological origin since it cannot be understood from the viewpoint of perturbation theory. Further discussions of the behaviors around the phase boundaries are given in the Appendix.

For $2 r_{w}<m_{0} \leqslant 4 r_{w}$, there are $d$ doubled modes each having only one nonzero $(\pi / a)$ momentum component which gives $u(p)<0$ and $T(p) \neq 0$; hence, they are restored to the theory and each one of them carries negative chiral charge. When we turn on the background gauge field with topological charge $Q_{\text {top }}$, these $d$ double modes produce an index $-d Q_{\text {top }}$ which adds to the index $+Q_{\text {top }}$ of the primary mode and thus gives the total index $(1-d) Q_{\text {top }}$. It agrees with numerical experiments for $D$ in smooth background gauge fields except at the phase boundary $m_{0}=4 r_{w}$, where the actual index is always zero.

For $4 r_{w}<m_{0} \leqslant 6 r_{w}$ (provided that $d \geqslant 4$ ), there are additional $d(d-1) / 2$ doubled modes restored to the theory. Each of them has only two nonzero momentum components which give $u(p)<0$ and $T(p) \neq 0$, and hence carries positive chiral charge. Then the total chiral charge of the theory is $1-d$ 
$+d(d-1) / 2$ after taking into account the primary mode, the $d$ doubled modes having one nonzero momentum component, and $d(d-1) / 2$ doubled modes having two nonzero momentum components. When we turn on the background gauge field with topological charge $Q_{\text {top }}$, the index is equal to (2 $\left.-3 d+d^{2}\right) Q_{\text {top }} / 2$. This is a topologically improper phase.

The process of dividing $m_{0}$ into topologically improper phases each of width $2 r_{w}$ continues until $m_{0}=2 d r_{w}$, then for $m_{0}>2 d r_{w}, T(p) \neq 0$ for all doubled modes, and they are all restored to the theory. The index must be zero for any gauge configurations: thus, the theory is topologically trivial for $m_{0}>2 d r_{w}$.

Now we can pull the exact solutions of the eigenvalues and fermion propagator together to obtain a complete picture of the topological phases in the free fermion limit. From the exact solutions of eigenvalues, Eq. (24), and the fermion propagator, Eqs. (28), (29), and (31), we immediately obtain that the +2 eigenmodes must have $T(p)=0$ in the fermion

$$
\operatorname{index}\left[D^{(k)}\right]=\left\{\begin{array}{c}
0 \\
\frac{(-1)^{k+1} d !}{d \Gamma(1+d-k) \Gamma(k)} Q_{\text {top }} \\
0
\end{array}\right.
$$

It is evident that Eq. (34) satisfies the exact reflection symmetry index $\left[D^{(k)}\right]=-$ index $\left[D^{(d+1-k)}\right]$ [equivalent to Eq. (17)] except at each one of the phase boundaries $\left(m_{0}\right.$ $\left.=2 k r_{w}, k=1, \ldots, d\right)$ where the index is nonzero and equal to the index on the LHS of the boundary. Then it is obvious that Eq. (34) is invalid at the symmetry axis $m_{0}=d r_{w}$ where the index must be zero, Eq. (18), according to the exact reflection symmetry. In general, we can prove that Eq. (34) breaks down at each one of the phase boundaries $\left(m_{0}=2 k r_{w}, k\right.$ $=1, \ldots, d)$.

Proof. Assuming Eq. (34) holds at the phase boundary $m_{0}=x$, then we have

$$
\text { index }[D(x)]=\operatorname{index}[D(x-\epsilon)] \text {, }
$$

where $\epsilon$ is an infinitesimal positive real number. Then the image of $m_{0}=x$ with respect to the mirror at $m_{0}=d r_{w}$ is $m_{0}^{\prime}=2 d r_{w}-x$, and its index is

$$
\operatorname{index}\left[D\left(2 d r_{w}-x\right)\right]=\operatorname{index}[D(x)]
$$

according to the exact reflection symmetry, Eq. (17). Now consider the image of $m_{0}=x-\epsilon$, and its index is

$$
\text { index }\left[D\left(2 d r_{w}-x+\epsilon\right)\right]=-\operatorname{index}[D(x-\epsilon)]
$$

Then from Eqs. (35)-(37), we obtain

$$
\text { index }\left[D\left(2 d r_{w}-x+\epsilon\right)\right]=\operatorname{index}\left[D\left(2 d r_{w}-x\right)\right] \text {. }
$$

propagator and thus are completely decoupled from the theory; while a zero mode has $T(p)=|u(p)|$ : hence its presence depends on the value of $m_{0}$. Therefore, using Eq. (23), from the total chiral charge of the zero modes, we can obtain a formula for the index of $D$ in a smooth background gauge field. From Eqs. (31), (32), we obtain the general formula for the total chiral charge of the zero modes in each phase:

$$
\begin{gathered}
Q_{5}^{(k)}=\sum_{i=1}^{k} \frac{(-1)^{i-1} d !}{(i-1) !(d-i+1) !}=\frac{(-1)^{k+1} d !}{d \Gamma(1+d-k) \Gamma(k)}, \\
k=1, \ldots, d,
\end{gathered}
$$

where the integer $k$ denotes the $k$ th phase region, 2( $k$ -1) $r_{w}<m_{0} \leqslant 2 k r_{w}$, which includes the upper phase boundary, and $Q_{5}=0$ for $m_{0} \leqslant 0$ and $m_{0}>2 d r_{w}$. Inserting Eq. (33) into Eq. (23), we obtain the index of $D$ in each phase as the following:

$$
\begin{gathered}
\text { if } k=0 \text { for } m_{0} \leqslant 0, \\
\text { if } k=1, \ldots, d \text { for } 2(k-1) r_{w}<m_{0} \leqslant 2 k r_{w}, \\
\text { if } k=d+1 \text { for } m_{0}>2 d r_{w} .
\end{gathered}
$$

This leads to a contradiction since the indices of two neighboring phases must be different. Therefore Eq. (35) must be invalid since the exact reflection symmetry is always true under any circumstances. This completes the proof that Eq. (34) fails at each one of the phase boundaries $\left(m_{0}=2 k r_{w}\right.$, $k=1, \ldots, d)$.

In Table I, we list the real eigenmodes of $D$ in the free fermion limit on a two-dimensional lattice with periodic boundary conditions. The lattice can be an infinite lattice or a finite lattice with an even number of sites in each dimension. First, we recall that the zero modes and +2 eigenmodes of $D$ are both chiral: [3] thus, their chiral charges can be determined by the number of nonzero $(\pi / a)$ momentum components of this fermion mode. We also recall that for each real eigenvalue of $D$ in two dimensions, it has degeneracy 2, which consists of a pair of eigenmodes of \pm 1 chiralities, while complex eigenvalues must come in complex conjugate pairs with chirality zero. The chirality degeneracy of real eigenmodes is not shown explicitly in Table I. In the first phase $-\infty<m_{0} \leqslant 0$, there are no zero modes and the theory in fact does not describe a massless fermion. The factor $T(p)$ is zero at the origin $p=0$ as well as at the corners of the Brillouin zone. In the second phase $0<m_{0}<2 r_{w}$, there is one zero mode with positive chiral charge. The theory in this phase correctly describes a single flavor of massless fermions. At the phase boundary $m_{0}=2 r_{w}$, two +2 real eigenmodes and their chirality partners become two pairs of complex conjugate eigenmodes $(1 \pm i)$ which are decoupled from the theory, i.e., $T(p)=0$. The number of zero modes remains 
TABLE I. The real eigenmodes of $D$ in the free fermion limit on two-dimensional lattice with periodic boundary conditions. The first column lists the ranges of $m_{0}$ values, the second column the real eigenvalues, the third column the multiplicities of the eigenvalues in momentum space, the fourth column the effective $\gamma_{5}$ of the eigenmode where the sign of $\gamma_{5}$ is used to denote the chiral charge of the eigenmode, and the last column is the total chiral charge of the zero modes. These results are obtained analytically from exact solutions on a finite lattice of an even number of sites in each dimension or the infinite lattice.

\begin{tabular}{lcccc}
\hline \hline & $\begin{array}{c}\text { Real } \\
\text { eigenvalues }\end{array}$ & Multiplicity & $\gamma_{5}^{\prime}$ & $\begin{array}{c}\text { Total chiral } \\
\text { charge of } \\
\text { zero modes }\end{array}$ \\
\hline$-\infty<m_{0} \leqslant 0$ & 2 & 2 & $+\gamma_{5}$ & \\
$0<m_{0}<2 r_{w}$ & 2 & 2 & $-\gamma_{5}$ & 0 \\
& 2 & 1 & $+\gamma_{5}$ & \\
$m_{0}=2 r_{w}$ & 2 & 2 & $-\gamma_{5}$ & \\
$2 r_{w}<m_{0}<4 r_{w}$ & 0 & 1 & $+\gamma_{5}$ & 1 \\
& 2 & 1 & $+\gamma_{5}$ & \\
$m_{0}=4 r_{w}$ & 0 & 1 & $+\gamma_{5}$ & 1 \\
$4 r_{w}<m_{0}<\infty$ & 0 & 1 & $+\gamma_{5}$ & \\
& 0 & 1 & $+\gamma_{5}$ & \\
\hline \hline
\end{tabular}

the same. These two pairs of complex conjuagte eigenmodes become two zero modes (in chirality pairs) in the third phase $2 r_{w}<m_{0}<4 r_{w}$. At the phase boundary $m_{0}=4 r_{w}$, the number of zero modes remains the same, but the +2 eigenmode (and its chirality partner) become a complex conjugate pair $(1 \pm i)$. Then they reappear as a zero mode (in a chirality pair) in the last phase $4 r_{w}<m_{0}<\infty$, in which all real eigenmodes are zero modes. We notice the interplay between the zero modes and the +2 eigenmodes when we change the value of $m_{0}$ from $-\infty$ to $+\infty$. Only in the region $0<m_{0}$ $<2 r_{w}$ and the critical point $m_{0}=2 r_{w}$ does the theory have the correct particle content to describe a single flavor of massless fermions, while other regions suffer from either species doubling or the absence of zero modes. The total chiral charge of zero modes in each phase is listed in the last column, from which we can predict the index of $D$ in a smooth background gauge field according to Eq. (23), i.e., Eq. (34). As we will see, when we turn on a smooth background gauge field with nonzero topological charge, Eq. (34) is satisfied exactly for all phases except at the phase boundaries where the index is always zero regardless of the total chiral charge (see Table III).

In Table II, we list the real eigenmodes of $D$ in the free fermion limit on a four-dimensional lattice with periodic boundary conditions. Although it has many more phases than Table I for two dimensions, however, it shares the same essential features of Table I: namely, only the region $0<m_{0}$ $<2 r_{w}$ and the critical point $m_{0}=2 r_{w}$ can describe a single flavor of massless fermions. We also recall that for each real
TABLE II. The real eigenmodes of $D$ in the free fermion limit on a four-dimensional lattice with periodic boundary conditions. The first column lists the ranges of $m_{0}$ values, the second column the real eigenvalues, the third column the multiplicities of the eigenvalues in momentum space, the fourth column the effective $\gamma_{5}$ of the eigenmode where the sign of $\gamma_{5}$ is used to denote the chiral charge of the eigenmode, and the last column the total chiral charge of the zero modes. These results are obtained analytically from exact solutions on a finite lattice of an even number of sites in each dimension or the infinite lattice.

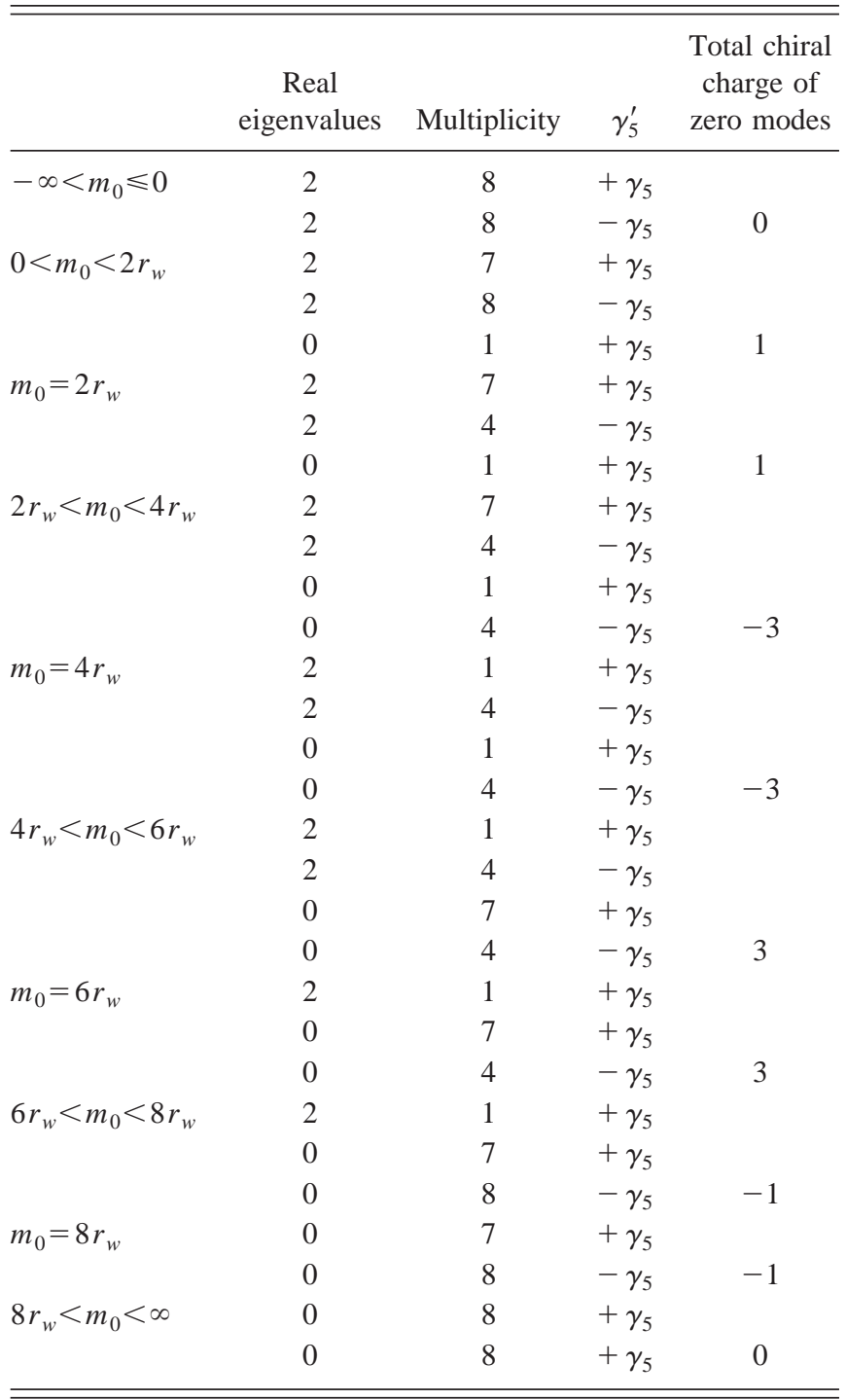

eigenvalue of $D$ in four dimensions, it has degeneracy 4, which consists of two pairs of eigenmodes of \pm 1 chiralities, while complex eigenvalues come in complex conjugate pairs with chirality 0 and degeneracy 2 . The chirality degeneracy of real eigenmodes is not shown explicitly in Table II. We will not go through each phase in Table II as we have done for Table I since it is straightforward to obtain these results from exact solutions of eigenvalues, Eq. (24), and the fermion propagator, Eqs. (28), (29), and (31).

Our next task is to turn on the background gauge field to see to what extent the formula, Eq. (34), which relies on weak coupling perturbation theory and the exact solutions in 
the free fermion limit, is satisfied in a background gauge field with nonzero topological charge, as well as to see the displacements of the phase boundaries with respect to the background gauge field.

\section{TOPOLOGICAL PHASE DIAGRAMS FOR $d=2$ AND $d=4$}

In this section we turn on the background gauge field to investigate topological phases of $D$ on two- and fourdimensional lattices, respectively. First, we define the background gauge fields in the following. On a four-dimensional torus $\left(x_{\mu} \in\left[0, L_{\mu}\right], \mu=1, \ldots, 4\right)$, the simplest nontrivial $\mathrm{SU}(2)$ gauge fields can be represented as

$$
\begin{aligned}
& A_{1}(x)=\tau\left[\frac{2 \pi h_{1}}{L_{1}}-\frac{2 \pi q_{1} x_{2}}{L_{1} L_{2}}+A_{1}^{(0)} \sin \left(\frac{2 \pi n_{2}}{L_{2}} x_{2}\right)\right], \\
& A_{2}(x)=\tau\left[\frac{2 \pi h_{2}}{L_{2}}+A_{2}^{(0)} \sin \left(\frac{2 \pi n_{1}}{L_{1}} x_{1}\right)\right], \\
& A_{3}(x)=\tau\left[\frac{2 \pi h_{3}}{L_{3}}-\frac{2 \pi q_{2} x_{4}}{L_{3} L_{4}}+A_{3}^{(0)} \sin \left(\frac{2 \pi n_{4}}{L_{4}} x_{4}\right)\right], \\
& A_{4}(x)=\tau\left[\frac{2 \pi h_{4}}{L_{4}}+A_{4}^{(0)} \sin \left(\frac{2 \pi n_{3}}{L_{3}} x_{3}\right)\right],
\end{aligned}
$$

where $\tau$ is one of the generators of $\mathrm{SU}(2)$ with the normalization $\operatorname{tr}\left(\tau^{2}\right)=2$, and $q_{1}$ and $q_{2}$ are integers. The global part is characterized by the topological charge

$$
Q=\frac{1}{32 \pi^{2}} \int d^{4} x \epsilon_{\mu \nu \lambda \sigma} \operatorname{tr}\left(F_{\mu \nu} F_{\lambda \sigma}\right)=2 q_{1} q_{2},
$$

which must be an integer. The harmonic parts are parametrized by four real constants $h_{1}, h_{2}, h_{3}$, and $h_{4}$. The local parts are chosen to be sinusoidal fluctuations with amplitudes $A_{1}^{(0)}, A_{2}^{(0)}, A_{3}^{(0)}$, and $A_{4}^{(0)}$, and frequencies $2 \pi n_{2} / L_{2}$, $2 \pi n_{1} / L_{1}, 2 \pi n_{4} / L_{4}$, and $2 \pi n_{3} / L_{3}$ where $n_{1}, n_{2}, n_{3}$, and $n_{4}$ are integers. The discontinuity of $A_{1}(x)\left[A_{3}(x)\right]$ at $x_{2}$ $=L_{2}\left(x_{4}=L_{4}\right)$ due to the global part only amounts to a gauge transformation. The field tensors $F_{12}$ and $F_{34}$ are continuous on the torus, while other $F$ 's are zero. To transcribe the continuum gauge fields to the lattice, we take the lattice sites at $x_{\mu}=0, a, \ldots,\left(N_{\mu}-1\right) a$, where $a$ is the lattice spacing and $L_{\mu}=N_{\mu} a$ is the lattice size. Then the link variables are

$$
U_{1}(x)=\exp \left[i A_{1}(x) a\right],
$$

$U_{2}(x)=\exp \left[i A_{2}(x) a+i \delta_{x_{2},\left(N_{2}-1\right) a} \frac{2 \pi q_{1} x_{1}}{L_{1}} \tau\right]$,

$U_{3}(x)=\exp \left[i A_{3}(x) a\right]$,

$U_{4}(x)=\exp \left[i A_{4}(x) a+i \delta_{x_{2},\left(N_{4}-1\right) a} \frac{2 \pi q_{2} x_{3}}{L_{3}} \tau\right]$.

In Table III, we list the real eigenmodes of $D$ on a $6 \times 6$
TABLE III. The index of $D$ on a $6 \times 6$ lattice in the background gauge field with topological charge $Q=1$ and other parameters $h_{1}$ $=0.5, h_{2}=0.6, A_{1}^{(0)}=0.7, A_{2}^{(0)}=0.8$, and $n_{1}=n_{2}=1$. The Wilson parameter $r_{w}$ is set to 1.0. We note that the gauge field shifts the critical points from their values in the free fermion limit. The reflection symmetry is satisfied exactly by checking that the indices are odd under the reflection with respect to the symmetry axis $m_{0}$ $=2.0$, that the displacement at the first phase boundary $\delta_{0}$ $=0.5472$ is equal to minus of the displacement $\delta_{2}=3.4528-4.0$ $=-0.5472$ at the third phase boundary, and that the location of the symmetry axis $m_{0}=2.0$ is invariant for any background gauge field. All critical points are accurate up to \pm 0.00005 except that the central critical point 2.0 is exact. The indices in the last column agree exactly with Eq. (34) except at the phase boundary $\left(m_{0}=2.0\right)$.

\begin{tabular}{lcccc}
\hline \hline & $\begin{array}{c}\text { Real } \\
\text { eigenvalues }\end{array}$ & Multiplicity & Chirality & Index \\
\hline$-\infty<m_{0} \leqslant 0.5472$ & 2 & 1 & +1 & 0 \\
$0.5473 \leqslant m_{0}<2.0$ & 0 & 1 & -1 & 1 \\
$m_{0}=2.0$ & 2 & 1 & -1 & 0 \\
$2.0<m_{0} \leqslant 3.4527$ & 0 & 1 & +1 & -1 \\
$3.4528 \leqslant m_{0}<\infty$ & & & & 0 \\
\hline \hline
\end{tabular}

lattice in the background gauge field with topological charge $Q=1$ and other parameters $h_{1}=0.5, h_{2}=0.6, A_{1}^{(0)}=0.7$, $A_{2}^{(0)}=0.8$, and $n_{1}=n_{2}=1$. The Wilson parameter $r_{w}$ has been set to 1.0. The indices of $D$ in the last column are computed by counting the zero modes according to index $(D)=n_{-}-n_{+}$. They agree exactly with the formula (34) with the total chiral charge of the zero modes obtained from the exact solutions in the free fermion limit as listed in the last column of Table I, except at the phase boundaries where the index is always zero regardless of the total chiral charge. The response of $D$ in the first region is easily understood since it has no zero modes in the free fermion limit and thus it cannot possess any zero modes after we turn on the gauge field with nonzero topological charge. The phase boundary has been shifted from $m_{0}=0$ to $m_{0}=0.5472$ due to the background gauge fields. The index of $D$ is always zero in this phase and $D$ is called topologically trivial. In the second phase, the index of $D$ is always equal to the topological charge of the gauge field, and $D$ is called topologically proper. Since $D$ is free of species doubling and the chiral charge of the zero modes is $+1, D$ satisfies the Atiyah-Singer index theorem exactly in this phase. However, at the phase boundary of the central critical line $m_{0}=2.0, D$ has the correct particle content in the free fermion limit, but it does not possess any zero modes after turning on the topologically nontrivial background gauge field. This cannot be understood from the viewpoint of perturbation theory, though we know that the index must be zero at the symmetry axis, Eq. (18), due to the exact reflection symmetry. Further discussions of the behaviors at the phase boundary are given in the Appendix. If we step into the next phase, we find that the index is opposite to the topological charge, in complete agreement with the formula (34) with total chiral charge 


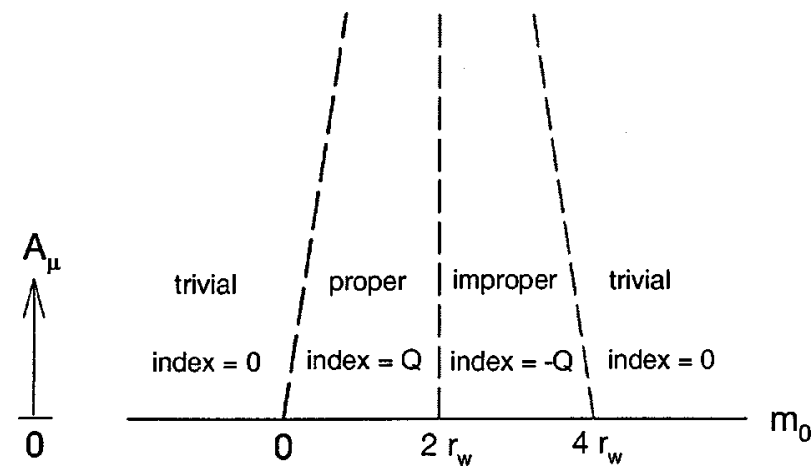

FIG. 2. The topological phases of $D$ on a two-dimensional lattice for smooth background gauge fields. The phase boundaries denoted by dashed lines are sketched to indicate their evolutions with respect to the background gauge field. The reflection symmetry with respect to the central critical line $m_{0}=2 r_{w}$ is displayed explicitly. Note that the central critical line $m_{0}=2 r_{w}$ is invariant for any background gauge configurations. The phase transition points in the free fermion limit (at the horizontal line) are determined exactly.

$Q_{5}=-1$ in the free fermion limit, as listed in Table I. In the last phase, $D$ has four species of massless fermion modes in the free fermion limit, similar to the case of the naive lattice fermion: then, there are no zero modes and the index is zero for any background gauge field. We note that the displacements of the phase boundaries due to the background gauge field must obey the exact reflection symmetry $\delta_{k}=-\delta_{d-k}$, $k=0, \ldots, d$, which is an immediate consequence of Eq. (17). In Table III, $\delta_{0}=0.5472$ at the first phase boundary is equal to minus the displacement $\delta_{2}=3.4572-4.0=-0.5472$ at the third phase boundary. The central phase boundary $m_{0}=2.0$ is invariant for any background gauge field. In Fig. 2, we sketch the topological phase diagram of $D$ in smooth background gauge fields on a two-dimensional lattice, which summarizes the gross features of a lot of numerical experiments. The discrete symmetry index $\left[D\left(4 r_{w}-m_{0}\right)\right]=$ - index $\left[D\left(m_{0}\right)\right]$ is satisfied exactly in all cases. The index in each phase agrees exactly with the formula (34), with the total chiral charge of the zero modes obtained from the exact solutions in the free fermion limit as listed in the last column of Table I, except at the phase boundaries where the index is always zero regardless of the total chiral charge.

In Table IV, we list the real eigenmodes of $D$ on a $4^{4}$ lattice in the background gauge field with topological charge $Q=2$ and other parameters $h_{1}=0.1, h_{2}=0.2, h_{3}=0.3, h_{4}$ $=0.4$, and $A_{1}^{(0)}=A_{2}^{(0)}=A_{3}^{(0)}=A_{4}^{(0)}=0$. The Wilson parameter $r_{w}$ is set to 1.0. There are more phase regions than those in two dimensions (see Table III), and the locations of all critical points are shifted by the background gauge field except the central critical point $m_{0}=4.0$. It is evident that the reflection symmetry, Eq. (17) is satisfied exactly. We note that the displacements of the phase boundaries due to the background gauge field are $\delta_{0}=0.3762, \delta_{1}=2.1881-2.0$ $=0.1881, \quad \delta_{2}=0, \quad \delta_{3}=5.8119-6.0=-0.1881, \quad$ and $\delta_{4}$ $=7.6238-8.0=-0.3762$, which satisfy the exact reflection symmetry $\delta_{k}=-\delta_{4-k}, k=0, \ldots, 4$. We do not go through detailed descriptions for each phase as we have done for Table III. We have also performed extensive numerical tests in the
TABLE IV. The index of $D$ on a $4^{4}$ lattice in the background gauge field with topological charge $Q=2$ and other parameters $h_{1}$ $=0.1, h_{2}=0.2, h_{3}=0.3, h_{4}=0.4$, and $A_{1}^{(0)}=A_{2}^{(0)}=A_{3}^{(0)}=A_{4}^{(0)}=0$. The reflection symmetry index $\left[D\left(8-m_{0}\right)\right]=-\operatorname{index}\left[D\left(m_{0}\right)\right]$ is satisfied exactly; in particular, the displacements of the phase boundaries due to the background gauge field satisfy the relationship $\delta_{k}=-\delta_{4-k}, k=0, \ldots, 4$. All critical points are accurate up to \pm 0.00005 except that the central critical point 4.0 is exact. The indices in the last column agree exactly with the formula (34), except at the phase boundaries $m_{0}=2.1881,4.0$, and 5.8119.

\begin{tabular}{lcccc}
\hline \hline & $\begin{array}{c}\text { Real } \\
\text { eigenvalues }\end{array}$ & Multiplicity & Chirality & Index \\
\hline$-\infty<m_{0} \leqslant 0.3762$ & & & & 0 \\
$0.3763 \leqslant m_{0} \leqslant 2.1880$ & 2 & 2 & +1 & \\
$m_{0}=2.1881$ & 0 & 2 & -1 & 2 \\
$2.1882 \leqslant m_{0}<4.0$ & 2 & 6 & -1 & 0 \\
$m_{0}=4.0$ & 0 & 6 & +1 & -6 \\
$4.0<m_{0} \leqslant 5.8118$ & 2 & 6 & +1 & 0 \\
$m_{0}=5.8119$ & 0 & 6 & -1 & 6 \\
$5.8120 \leqslant m_{0} \leqslant 7.6237$ & 2 & 2 & +1 & 0 \\
$7.6238 \leqslant m_{0}<\infty$ & 0 & 2 & -1 & -2 \\
\hline
\end{tabular}

topologically proper phase as well as in the improper phases. For smooth background gauge fields with nonzero topological charge, the exact zero modes with definite chirality are reproduced to a very high precision on a finite fourdimensional lattice, and the index theorem is satisfied exactly in the topologically proper phase. The zero modes are also very stable under random fluctuations as defined in Eq. (43) of Ref. [3]. This completes our intended investigations for $D$ in four-dimensional smooth background gauge fields as outlined in [3]. In Fig. 3, we sketch the topological phase diagram of $D$ in smooth background gauge fields on a fourdimensional lattice with an even number of sites in each dimension, which summarizes the gross features of a lot of numerical experiments. The discrete symmetry index $\left[D\left(8 r_{w}-m_{0}\right)\right]=-\operatorname{index}\left[D\left(m_{0}\right)\right]$ is satisfied exactly in all cases. The index in each phase agrees exactly with the formula (34) except at the phase boundaries where the index is always zero regardless of the total chiral charge.

It is interesting to note that in Ref. [9], the spectral flow of the Hermitian Wilson-Dirac operator $H_{w}\left(m_{0}\right)=\gamma_{5} D_{w}\left(m_{0}\right)$ is studied numerically for smooth $\mathrm{SU}(2)$ instanton backgrounds on an $8^{4}$ lattice, and the symmetry $-H_{w}(8$ $\left.-m_{0}, U\right)=H_{w}\left(m_{0},-U\right)$ is displayed. Since the index of $D$ can also be computed by studying the level crossings in the spectral flow of $H_{w}$ as a function of $m_{0}$ [see Eq. (A2) in the Appendix], the results of Ref. [9] provide another fourdimensional verification of the reflection symmetry (17) proved in Sec. II. Furthermore, in Ref. [10], the spectrum of the Hermitian Neuberger-Dirac operator $H=\gamma_{5} D$ in a smooth $\mathrm{SU}(2)$ instanton background is investigated in the phase $0<m_{0}<2$. The chiral properties of $D(H)$ are verified, 


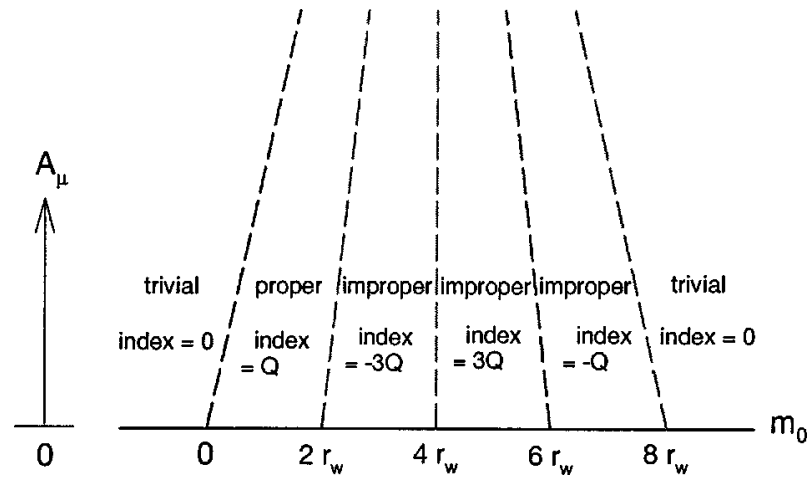

FIG. 3. The topological phases of $D$ on a four-dimensional lattice for smooth background gauge fields. The phase boundaries with dashed lines are sketched to indicate their evolutions with respect to the background gauge field. The reflection symmetry with respect to the central critical line $m_{0}=4 r_{w}$ is shown explicitly. Note that the central critical line $m_{0}=4 r_{w}$ is invariant for any background gauge configurations. The critical points in the free fermion limit (at the horizontal line) are determined exactly.

and the pole method [11] appears to be the most costeffective implementation.

\section{SUMMARY AND DISCUSSIONS}

We have investigated the topological phase diagrams of the Neuberger-Dirac operator $D$ with respect to the negative mass parameter $m_{0}$ and the background gauge field. When the gauge configurations are far from being rough, the phase diagrams can be schematically represented in Figs. 1, 2, and 3 for $d$ dimensions, two-dimensions, and four-dimensions, respectively. Each topological phase diagram (Figs. 1-3) possesses the exact reflection symmetry (17). The symmetry axis $m_{0}=d r_{w}$ is a phase boundary with zero index, and its position is invariant for any background gauge field. Among the many different phases in each phase diagram, there is only one phase in which the Atiyah-Singer index theorem is satisfied exactly, while other phases are either trivial or improper.

The formula (34) for the index of $D$ in each phase is derived by obtaining the total chiral charge of the zero modes in the exact solution of the free fermion propagator. For smooth gauge configurations, Eq. (34) is verified to be correct by numerical experiments on two- and fourdimensional finite lattices with an even number of sites in each direction, except at the phase boundaries starting at $\left(m_{0}=2 k r_{w}, k=1, \ldots, d\right)$ where the index is always zero for any background gauge fields. Moreover, using the exact reflection symmetry, we prove that Eq. (34) must break down at the phase boundaries. This indicates that there may be some nonperturbative or topological effects happening at the phase boundaries. Further discussions of the behaviors at the phase boundaries are given in the Appendix. Now it is natural to generalize Eq. (34) by incorporating the displacements of the phase boundaries due to the background gauge field and also imposing the index zero at all phase boundaries: then, Eq. (34) becomes

$$
\operatorname{index}\left[D^{(k)}\right]= \begin{cases}\frac{(-1)^{k+1} d !}{d \Gamma(1+d-k) \Gamma(k)} Q_{\text {top }} & \text { if } 2(k-1) r_{w}+\delta_{k-1}<m_{0}<2 k r_{w}+\delta_{k} \quad, \quad \text { for } k=1, \ldots, d, \\ 0 \quad \text { if } m_{0}=2 k r_{w}+\delta_{k} & \text { for } k=0, \ldots, d,\end{cases}
$$

where $\delta_{k}, k=0, \ldots, d$, are the displacements of the phase boundaries due to the background gauge field, which satisfy

$$
\delta_{k}=-\delta_{d-k} .
$$

Equations (48) and (49) constitute the general formula for the index of the Neuberger operator in a smooth background gauge field, as a function of the negative mass parameter $m_{0}$. The exact reflection symmetry (17) is obviously satisfied by Eqs. (48) and (49).

It is tempting to go beyond the limit of smooth background gauge fields by increasing the field strength or its roughness, in order to explore what lies ahead in the upper portions of these phase diagrams. However, we quickly run into difficulties as the phase boundaries bifurcate into many different phases, reminiscent of the chaos in dynamical systems. Even for $D$ in a topologically proper phase an infinitesimal change in $m_{0}$ may end up having $D$ in a trivial or improper phase. For a given rough gauge configuration, we do not know a priori what the range of $m_{0}$ values is for $D$ to be in the topologically proper phase. We can easily demonstrate the bifurcation of the topological phases of $D$ by increasing the amplitudes of the local sinusoidal fluctuations of the background gauge field on a two-dimensional lattice. In Table V, all parameters are the same as in Table III except that the amplitude $A_{1}^{(0)}$ is increased from 0.7 to 2.0. We see that there are a total of 13 phase regions which result from bifurcations in two steps. It is evident that formula (48) fails completely. However, the exact reflection symmetry, Eq. (17), for the index in each phase, as well as the positions of the phase boundaries, is still satisfied exactly, though we cannot predict the number of different topological phases and the index of $D$ in each phase. We do not know precisely the conditions under which the bifurcations may happen, but one thing for sure is that the index is identically zero at all phase boundaries.

We have also measured the indices of $D$ with $m_{0}$ fixed at 1.0 for an ensemble of gauge configurations from quenched simulations and compared them to the geometrically defined 
TABLE $\mathrm{V}$. The index of $D$ on a $6 \times 6$ lattice in the background gauge field with topological charge $Q=1$ and other parameters $h_{1}$ $=0.5, h_{2}=0.6, A_{1}^{(0)}=2.0 . A_{2}^{(0)}=0.8$, and $n=n_{2}=1$. The Wilson parameter $r_{w}$ is set to 1.0. The topological phases have bifurcated from 4 into 13 regions in two steps. The reflection symmetry with respect to the central critical line $m_{0}=2.0$ is satisfied exactly by the indices in all phases as well as the positions of the critical points. All critical points are accurate up to \pm 0.00005 except that the central critical point 2.0 is exact.

\begin{tabular}{|c|c|c|c|c|}
\hline & $\begin{array}{c}\text { Real } \\
\text { eigenvalues }\end{array}$ & Multiplicity & Chirality & Index \\
\hline$-\infty<m_{0} \leqslant 0.7002$ & & & & 0 \\
\hline \multirow[t]{2}{*}{$0.7003 \leqslant m_{0} \leqslant 0.9050$} & 2 & 1 & +1 & \\
\hline & 0 & 1 & -1 & 1 \\
\hline $0.9051 \leqslant m_{0} \leqslant 0.9662$ & & & & 0 \\
\hline \multirow{2}{*}{$0.9663 \leqslant m_{0} \leqslant 1.4545$} & 2 & 1 & -1 & \\
\hline & 0 & 1 & +1 & -1 \\
\hline $1.4546 \leqslant m_{0} \leqslant 1.5592$ & & & & 0 \\
\hline \multirow[t]{2}{*}{$1.5593 \leqslant m_{0} \leqslant 2.0$} & 2 & 1 & -1 & \\
\hline & 0 & 1 & +1 & -1 \\
\hline$m_{0}=2.0$ & & & & 0 \\
\hline \multirow[t]{2}{*}{$2.0<m_{0} \leqslant 2.4407$} & 2 & 1 & +1 & \\
\hline & 0 & 1 & -1 & +1 \\
\hline $2.4408 \leqslant m_{0} \leqslant 2.5454$ & & & & 0 \\
\hline \multirow[t]{2}{*}{$2.5455 \leqslant m_{0} \leqslant 3.0337$} & 2 & 1 & +1 & \\
\hline & 0 & 1 & -1 & +1 \\
\hline $3.0338 \leqslant m_{0} \leqslant 3.0949$ & & & & 0 \\
\hline \multirow[t]{2}{*}{$3.0950 \leqslant m_{0} \leqslant 3.2997$} & 2 & 1 & -1 & \\
\hline & 0 & 1 & +1 & -1 \\
\hline $3.2998 \leqslant m_{0}<\infty$ & & & & 0 \\
\hline
\end{tabular}

topological charges of the gauge configurations. It turns out that the index does not always agree with the geometrically defined topological charge. The discrepancies may be due to the fact that the fixed value $m_{0}=1.0$ does not fall into the topologically proper phase for all gauge configurations. If that is the case, we can proceed in the following way: for a given gauge configuration, we measure the index of $D\left(m_{0}\right)$ for sufficiently sampled $m_{0}$ values and exploit the reflection symmetry: then, we should be able to identify the correct index unambiguously. Otherwise, we must resolve the discrepancies from the fundamental viewpoint, namely, the very definition of topological charge on a lattice.

Finally we would like to remark that it is interesting to understand the underlying dynamics which provoke the collective motion of the eigenvalues of $D$ (or equivalently the zero crossings of the spectral flow of the Hermitian WilsonDirac operator $H_{w}=\gamma_{5} D_{w}$ ) at the phase boundaries (except for the first and last phase boundaries) such that the index always turns out to be zero, though the exact refection symmetry must impose the zero index at the phase boundary $m_{0}=d r_{w}$.

\section{APPENDIX}

In this appendix, we discuss the spectral flow of the Neuberger operator as a function of the negative mass parameter $m_{0}$, in particular its behaviors at the phase boundaries, in order to gain a perspective on the underlying mechanism which gives the zero index at the phase boundaries. Note that the index at the phase boundary $m_{0}=d r_{w}$ (the symmetry axis) must be zero due to the exact reflection symmetry (17). Since the Neuberger operator can be written in terms of the Hermitian Wilson-Dirac operator $H_{w}\left(m_{0}\right)=\gamma_{5} D_{w}\left(m_{0}\right)$,

$$
D=1+V=1+\gamma_{5} \frac{H_{w}}{\sqrt{H_{w}^{2}}},
$$

the index of $D$ is

$$
\text { index }(D)=\frac{1}{2} \operatorname{Tr}\left(\gamma_{5} V\right)=\frac{1}{2} \operatorname{Tr}\left(\frac{H_{w}}{\sqrt{H_{w}^{2}}}\right)=\frac{1}{2}\left(h_{+}-h_{-}\right),
$$

where "Tr" denotes the trace over the Dirac space and the position space, and $h_{+}\left(h_{-}\right)$is the number of positive (negative) eigenvalues of $H_{w}$. So the index of $D$ can be obtained by studying the level crossings of the low-lying eigenvalues of $H_{w}$ [9]. However, the spectral flow of the Neuberger operator $D$ seems to be more complicated than that of the Hermitian Wilson-Dirac operator $H_{w}$. Since eigenvalues of $H_{w}\left(m_{0}\right)$ are real, its spectral flow as a function of $m_{0}$ can be plotted as curves on a plane. On the other hand, the eigenvalues of the Neuberger operator are complex, lying in a unit circle with the center at 1 on the complex plane [3]; therefore, the spectral flow as a function of $m_{0}$ can be plotted as curves on the unit cylinder with center at 1 in the threedimensional space formed by the complex plane of the eigenvalues and parameter $m_{0}$. The relationship between the eigenvalues of $D$ and $H_{w}$ can be written as

$$
\lambda=1+U^{\dagger} \gamma_{5} U_{H} \frac{\lambda_{H}}{\sqrt{\lambda_{H}^{2}}} U_{H}^{\dagger} U,
$$

where $\lambda\left(\lambda_{H}\right)$ is the diagonal matrix of eigenvalues of $D$ $\left(H_{w}\right), U\left(U_{H}\right)$ is the unitary transformation matrix formed by the eigenfunctions, satisfying

$$
\begin{gathered}
D=U \lambda U^{\dagger}, \\
H_{w}=U_{H} \lambda_{H} U_{H}^{\dagger} .
\end{gathered}
$$

It is evident that the spectral flow of $D$ not only depends on the spectral flow of $H_{w}$, but also the overlap of their eigenfunctions.

In general, due to the limited precision of most numerical computations, one cannot trace the spectral flow of $D$ to any precision one wishes. However, even if one can trace the spectral flow to a very high precision, one still does not understand why the eigenvalues behave in such a manner at the phase boundaries, so that the index must be zero, except for the first and last phase boundaries. For example, let us describe the spectral flow of $D$ around the phase boundary $m_{0}=2$ in Table III. Although we know that its index must be zero due to the exact reflection symmetry, it is still instructive to examine the spectral flow around this phase boundary. 
At $m_{0}=2-\epsilon$, where $\epsilon$ is any positive infinitesimal number, the zero mode of negative chirality is accompanied by a +2 eigenmode of positive chirality, and the rest of the eigenvalues are in complex conjugate pairs. However, at $m_{0}=2$, the zero mode becomes a member of a complex conjugate pair $\left(a_{1} \pm i a_{2}\right)$, while the associated +2 eigenmode becomes a member of another complex conjugate pair $\left(2-a_{3} \pm i a_{4}\right)$, where $a_{i}(i=1, \ldots, 4)$ are positive small numbers. Presumably these two pairs of complex conjugates have no direct correlations since they are far apart. However, in order to create one additional complex conjugate pair out of the zero mode and its associated +2 real eigenmode, all other complex conjugate pairs must undergo a collective motion in the unit circle such that all eigenvalues turn out to be in complex conjugate pairs. Then, at $m_{0}=2+\epsilon$, all complex conjugate pairs again undergo another collective motion to yield a zero mode of positive chirality and its associated +2 eigenmode of negative chirality. It seems to be unlikely that such collective motions of the eigenvalues at the phase boundaries can be described by any perturbation theory in $m_{0}$ or the background gauge fields.

Now let us turn to the spectral flow of the Hermitian Wilson-Dirac operator $H_{w}=\gamma_{5} D_{w}$, which corresponds to the spectral flow of $D$ described above. At $m_{0}=2-\epsilon$, the eigenvalues of $H_{w}$ are mostly in plus-minus pairs except two positive small eigenvalues. Thus $h_{+}-h_{-}=2$, and the index of $D$ is one according to Eq. (A2). At $m_{0}=2$, one of the positive small eigenvalues crosses zero $^{2}$ and becomes a negative small eigenvalue. The transition is exactly at the

${ }^{2}$ If one examines the crossing numerically, one may find a seemingly discontinuity of order $10^{-8}$, however, which is only due to the limited precision of one's numerical computation. point $m_{0}=2$. So $h_{+}=h_{-}$, and the index is zero. Then at $m_{0}=2+\epsilon$, the remaining positive small eigenvalue also crosses zero and turns into a negative small eigenvalue. Since $h_{-}-h_{+}=2$, the index is -1 . Now the seeming puzzle is why these two positive small eigenvalues do not simultaneously become two negative small eigenvalues at the point $m_{0}=2$; then, the phase boundary of zero index would not exist and the formula (34) would also hold for the phase boundaries. However, this would lead to a contradiction to the exact reflection symmetry (18), which must hold in general, and the only way out is to have the spectral flow produce a zero index at the phase boundary of the symmetry axis $\left(m_{0}=d r_{w}\right)$. So this puzzle seems to be resolved, but the underlying mechanism is still not understood. In general, for phase boundaries other than the first one (stemming at $m_{0}$ $=0$ ), the symmetry axis $m_{0}=d r_{w}$, and the last one (stemming at $m_{0}=2 d r_{w}$ ), we do not have any arguments to explain why there must be a number $\frac{1}{2}\left|h_{+}-h_{-}\right|$of eigenvalues undergoing a sign-flipping transition when approaching one side of the phase boundary, while the number $\frac{1}{2}\left|h_{+}^{\prime}-h_{-}^{\prime}\right|$ is the other side of the phase boundary, such that the index must be zero at the phase boundary. Furthermore, it seems to be more difficult to tackle the underlying dynamics that provokes such transitions at the phase boundaries.

\section{ACKNOWLEDGMENTS}

I would like to thank Shailesh Chandrasekharan for drawing my attention to a remark in his paper [4]. After this paper was posted to the archive hep-lat, Chandrasekharan's results on the Schwinger model [12] were also posted. I also wish to thank Martin Lüscher for his comment in a correspondence. Finally, I thank Herbert Neuberger for his helpful discussion on the level crossings of the Hermitian Wilson-Dirac operator. This work was supported by the National Science Council, R.O.C., under grant NSC88-2112-M002-016.
[1] H. Neuberger, Phys. Lett. B 417, 141 (1998).

[2] H. Neuberger, Phys. Lett. B 427, 353 (1998).

[3] T. W. Chiu, Phys. Rev. D 58, 074511 (1998).

[4] S. Chandrasekharan, Phys. Rev. D 60, 074503 (1999).

[5] T. W. Chiu and T. H. Hsieh, "Perturbation calculation of the axial anomaly of Ginsparg-Wilson fermion,'” hep-lat/9901011.

[6] T. W. Chiu, Phys. Lett. B 445, 371 (1999).

[7] T. W. Chiu, Nucl. Phys. B (Proc. Suppl.) 73, 688 (1999).
[8] C. R. Gattringer, I. Hip, and C. B. Lang, Nucl. Phys. B508, 329 (1997).

[9] R. Edwards, U. Heller, and R. Narayanan, Nucl. Phys. B522, 285 (1998).

[10] R. Edwards, U. Heller, and R. Narayanan, Nucl. Phys. B540, 457 (1999).

[11] H. Neuberger, Phys. Rev. Lett. 81, 4060 (1998).

[12] S. Chandrasekharan, Phys. Rev. D 59, 094502 (1999). 